The Astrophysical Journal, 477:623-630, 1997 March 10

(C) 1997. The American Astronomical Society. All rights reserved. Printed in U.S.A.

\title{
A COMPARISON OF RADIO AXIS WITH HOST GALAXY PLANE AXIS IN SEYFERT GALAXIES
}

\author{
Henrique R. Schmitt, ${ }^{1,2,3}$ AnNe L. Kinney, ${ }^{1}$ Thaisa Storchi-BergmanN, ${ }^{2}$ AND \\ ROBERT ANTONUCCI ${ }^{4}$ \\ Received 1996 July 23; accepted 1996 October 4
}

\begin{abstract}
We use the radio axis as an indicator of the orientation of the obscuring torus in Seyfert galaxies and analyze the difference between the position angles of extended radio structures and host galaxy major axis of Seyfert 1 and Seyfert 2 galaxies. We find that Seyfert 1's are less likely to have extended radio structures along the host galaxy major axis while Seyfert 2's have these structures distributed in most directions. We also find a zone of avoidance in the distribution of position angles: both Seyfert 1's and Seyfert 2's seem to avoid close alignment between the radio axis and the host galaxy plane axis. These results are analyzed from the point of view of a model in which Seyfert 1's have their obscuring torus axis aligned preferentially along the host galaxy disk axis and Seyfert 2's have their torus axis lying at an intermediate angle between the galaxy disk and its axis.
\end{abstract}

Subject headings: galaxies: nuclei — galaxies: Seyfert — galaxies: structure — radio continuum: galaxies

\section{INTRODUCTION}

Since the discovery by Antonucci \& Miller (1985) of polarized broad emission lines in the nuclear spectrum of NGC 1068, interest in the unified model for Seyfert galaxies has grown considerably (see Antonucci 1993 and Urry \& Padovani 1995 for a review of the model). This model assumes that Seyfert 1's and Seyfert 2's both contain an active nucleus surrounded by a dusty torus and that the angle through which the central engine is observed determines the classification of the object. Several pieces of evidence corroborate this model, like the observation of polarized broad emission lines in several Seyfert 2 galaxies (Miller \& Goodrich 1990), the deficit of ionizing photons in Seyfert 2's (Wilson, Ward, \& Haniff 1988; Kinney et al. 1991), and the collimation of the nuclear radiation, observed as extended linear radio sources (Ulvestad \& Wilson 1989) and conically shaped narrow-line regions (NLRs; Pogge 1989).

In a recent paper, Schmitt \& Kinney (1996) studied the NLR shape of Seyfert galaxies, as predicted by the unified model, using archival high-resolution Hubble Space Telescope $[\mathrm{O} \mathrm{III}]$ images. Their results show that the sizes of Seyfert 1 galaxies' NLRs are much smaller than the NLRs of Seyfert 2's would be if they were observed pole-on. The sample was not selected by an isotropic property, but the similarity in radio and $5007 \AA \AA$ luminosities of Seyfert 1's and Seyfert 2's suggests that the comparison may be rather fair anyway. This result apparently contradicts the unified model, from which we would expect both kinds of objects to have similar intrinsic NLR sizes. In order to solve this problem, Schmitt \& Kinney (1996) propose that the Seyfert 1 's torus axis may be aligned preferentially along the host

\footnotetext{
${ }^{1}$ Space Telescope Science Institute, 3700 San Martin Drive, Baltimore, MD 21218; schmitt@stsci.edu, kinney@stsci.edu.

2 Departamento de Astronomia, Instituto de Física, Universidade Federal do Rio Grande do Sul, C.P. 15051, CEP 91501-970, Porto Alegre, RS, Brazil; thaisa@sofia.if.ufrgs.br.

${ }^{3} \mathrm{CNPq}$ Fellow.

${ }^{4}$ Department of Physics, University of California, Santa Barbara, Santa Barbara, CA 93106; antonucci@physics.ucsb.edu.
}

galaxy plane axis while the Seyfert 2's torus axis may lie at an intermediate angle between the galaxy plane axis and the galaxy plane. In this picture, because the amount of extended gas intercepted by the nuclear radiation is smaller perpendicular to the plane than at directions closer to it, the NLR appears more extended in Seyfert 2's than in Seyfert 1's.

The reason for the Seyfert 1 orientation distribution may be that even those objects that are seen from the polar nuclear torus direction, but nearly edge-on to the host galaxy, are classified as Seyfert 2 because of obscuration by dust in the host galaxy plane. There is ample evidence that the broad-line region in edge-on galaxies is reddened (de Zotti \& Gaskell 1985) or entirely extinguished (Keel 1980; Lawrence \& Elvis 1982).

In order to check the orientation of the torus axis in Seyfert 1's and Seyfert 2's, in this paper we compare the position angle (P.A.) of the extended nuclear radio structures of Seyfert 1's and Seyfert 2's with their host galaxies' major-axis P.A. We show that there is a lack of Seyfert 1's with radio structures aligned along the host galaxy major axis (which would correspond to the case in which the obscuring torus is nearly perpendicular to the galaxy plane) while Seyfert 2's have radio structures distributed along almost all directions, consistent with the model proposed by Schmitt \& Kinney (1996).

\section{THE SAMPLE AND MEASUREMENTS}

We selected from the literature a sample of 46 Seyfert galaxies (15 Seyfert 1's and 31 Seyfert 2's) having highresolution radio maps and showing linear or slightly resolved radio structures, as defined by Ulvestad \& Wilson (1984a). In Table 1, we give the galaxy names together with their activity type (Seyfert 1 or Seyfert 2), morphological type, mean numerical index $(T)$ of stage along the Hubble sequence (as defined in de Vaucouleurs et al. 1991), distance, $6 \mathrm{~cm}$ flux, logarithm of the $6 \mathrm{~cm}$ power, P.A. of the radio structure (P.A..$_{\text {rad }}$ ), P.A. of major axis (P.A.MA), the difference between P.A.rad and P.A.MA ( $\Delta$ P.A.), and the inclination of the host galaxy. The distances were calculated using the 


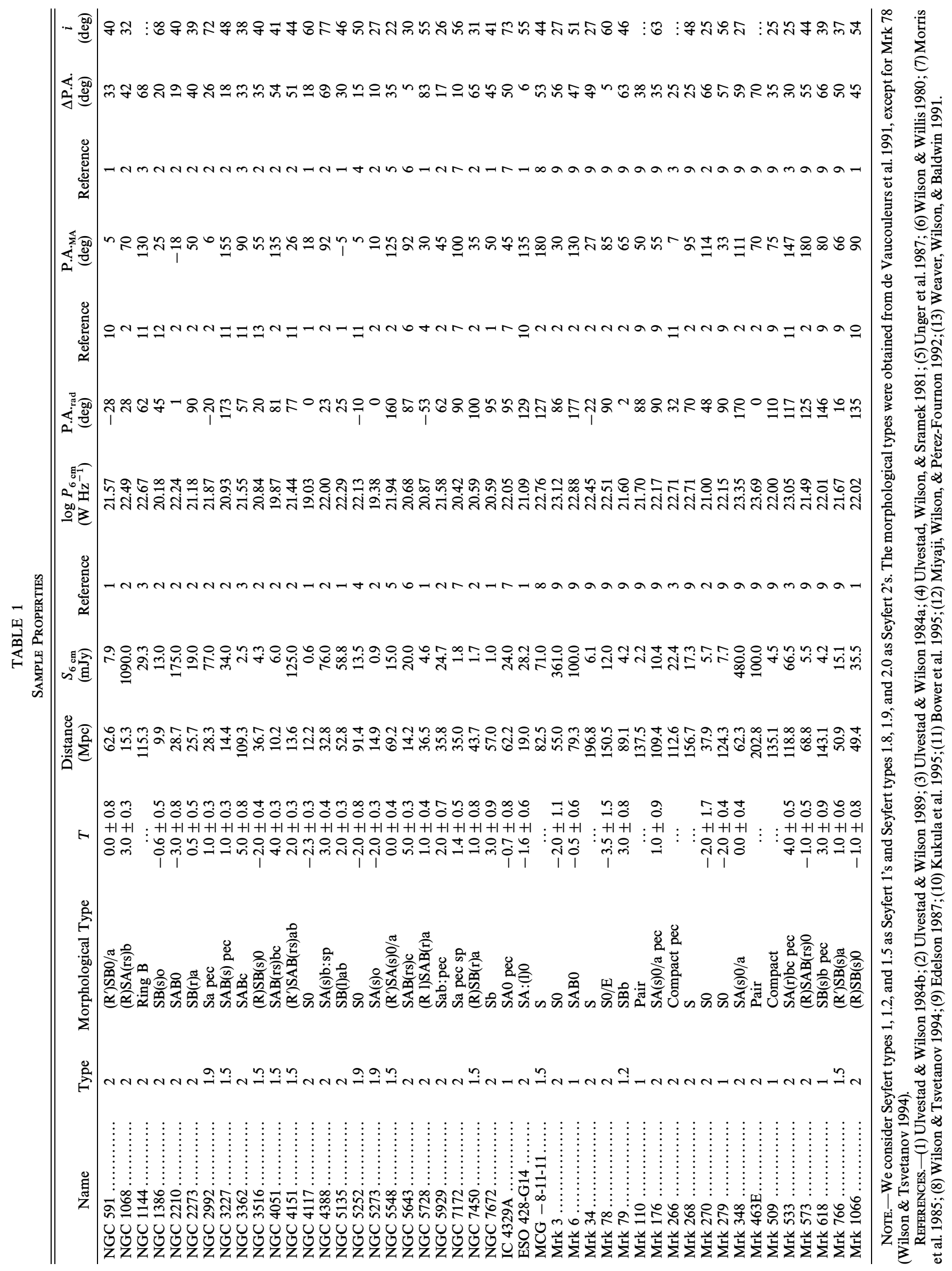




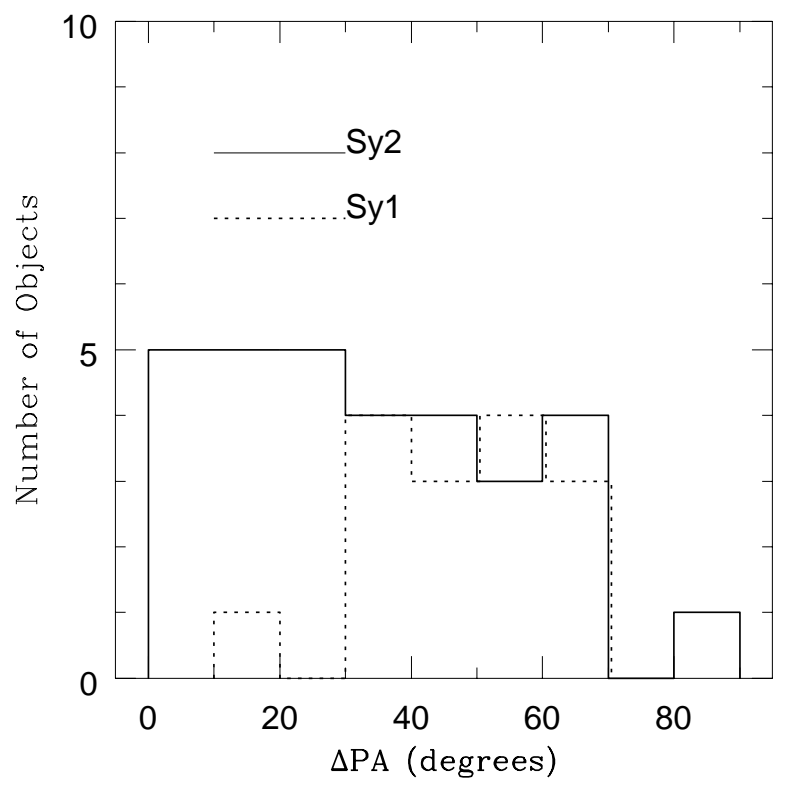

FIG. 1.- Histogram of the difference between the radio and the host galaxy major-axis position angles of Seyfert 1's (dashed line) and Seyfert 2's (solid line).

galaxies' radial velocities relative to the Local Group (de Vaucouleurs et al. 1991) and $H_{0}=75 \mathrm{~km} \mathrm{~s}^{-1} \mathrm{Mpc}^{-1}$. The inclinations were calculated from the axial ratios, assuming that the face-on galaxy is basically circular in shape.

The P.A.'s of the extended radio emission were obtained from the references in Table 1, using their published values or measuring it on their radio maps when the P.A. was not given explicitly. The host galaxies' major-axis P.A.'s were mostly obtained from de Vaucouleurs et al. (1991), with the remainder from references cited in Table 1. The P.A.'s were checked by looking at the galaxies on the Digitized Sky Survey plates. For the galaxies without values for the major-axis P.A. available in the literature and for the cases

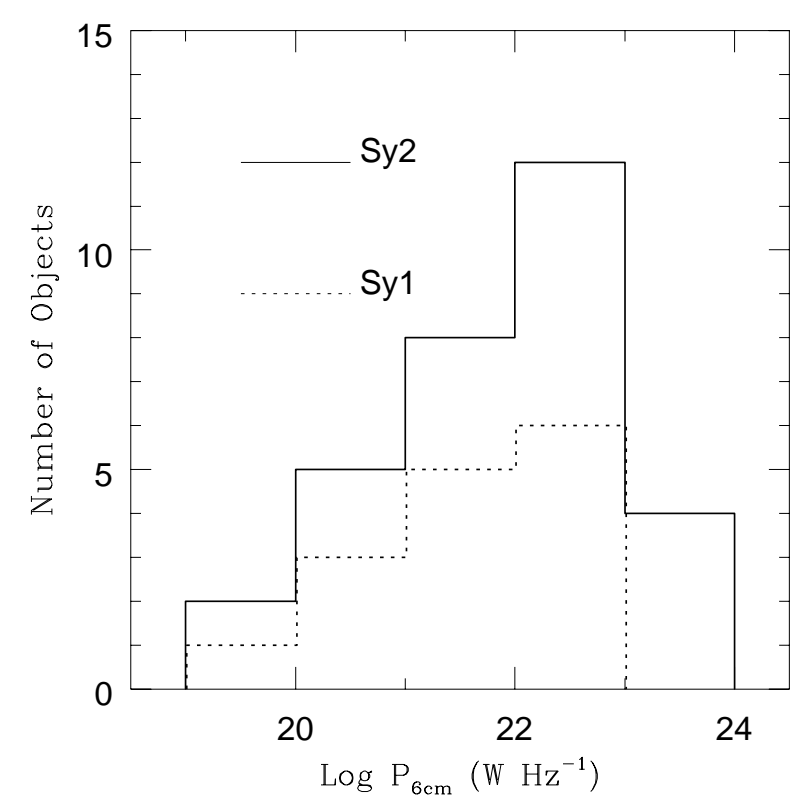

Fig. 2.-Distribution of the logarithm of the $6 \mathrm{~cm}$ radio power. Lines are as in Fig. 1.

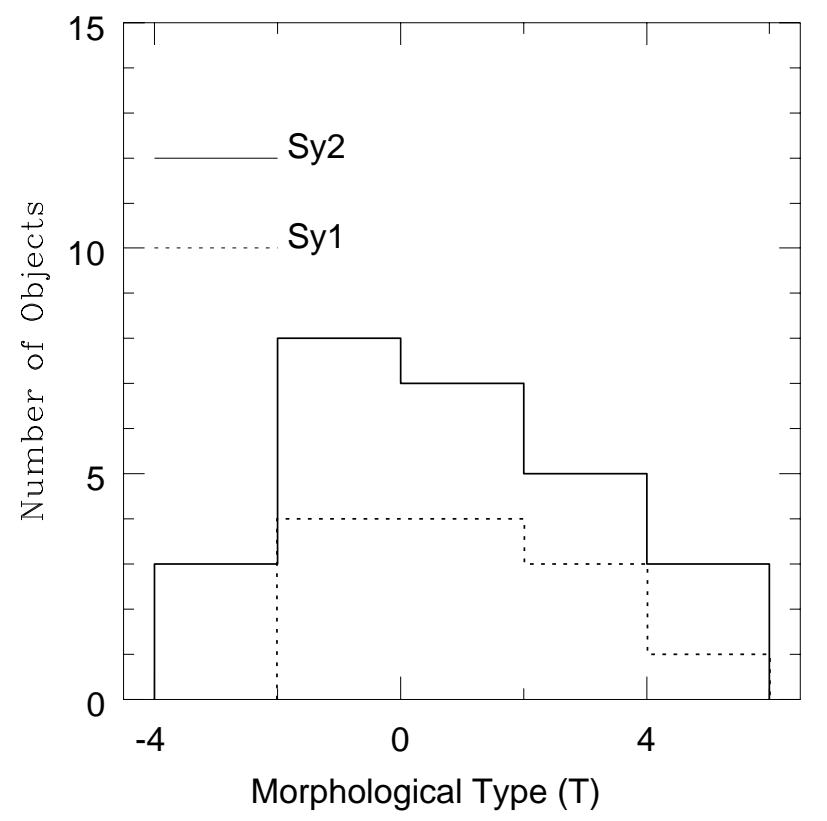

FIG. 3.-Distribution of the galaxies' morphological types $(T)$. Lines are as in Fig. 1. $T=-4$ corresponds to morphological type E, $T=-2$ corresponds to $\mathrm{S} 0, T=0$ corresponds to $\mathrm{S} 0 / \mathrm{a}, T=2$ corresponds to Sab, and $T=4$ corresponds to Sbc.

in which the published value was wrong (NGC 5929, Mrk 573, MCG -8-11-11), the P.A. was measured by fitting ellipses over the outer isophotes of the digitized galaxy image.

\section{RESULTS}

Figure 1 shows the histogram of the differences between the P.A.'s of the radio axis and of the host galaxy major axis. We can see that there is a lack of small values for Seyfert 1's, indicating that they are less likely to have extended radio structures along the host galaxy major axis.

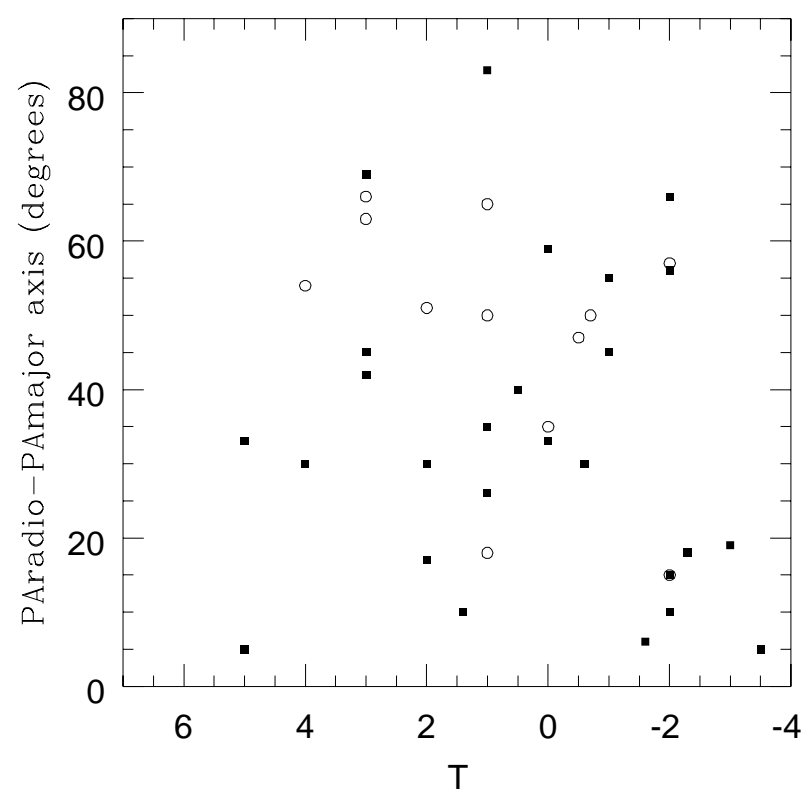

FIG. 4.-Comparison between the radio axis and major-axis position angle differences and the host galaxy morphological type. Circles are Seyfert 1's, and squares are Seyfert 2's. 
In other words, the cases in which the radio axis lies in the plane of the host galaxy are rare in Seyfert 1's, as expected. Meanwhile, the Seyfert 2's have $\Delta$ P.A. values evenly distributed from $0^{\circ}$ to $70^{\circ}$. However, it is interesting to note that both distributions have a lack of objects with radio structures well aligned with the host galaxy minor axis. Applying a Kolmogorov-Smirnov test to the data, we find that the hypothesis that the two groups of galaxies are drawn from the same parent population is rejected at the $99.0 \%$ level, or 99.1\% when we exclude double-nucleus galaxies (NGC 1144, Mrk 110, Mrk 266, and Mrk 463E).

We must ask whether the Seyfert 1's and 2's in the sample have similar intrinsic properties, or if the above results might be traceable to selection effects. First we compare the logarithm of the $6 \mathrm{~cm}$ radio power of the two groups, to see if they are similar in the two groups of galaxies, since the more luminous objects might be expected to have larger radio structures, which are easier to detect and measure. In Figure 2, we show the histogram of the logarithm of the 6 $\mathrm{cm}$ radio power, where we can see that both groups have very similar distributions of radio powers, except for the high-luminosity tail of Seyfert 2's. This high-luminosity tail has already been observed by Meurs \& Wilson (1984) and Ulvestad \& Wilson (1989). However, these objects are undistinguished in the position angle histograms, so they do not create the claimed effects. Note that the former reference includes disk emission, which may be significant in lowluminosity objects.

Wilson \& Tsvetanov (1994) have recently proposed that the obscuring torus axis may be aligned with the galaxy plane axis in late-type galaxies while the obscuring torus could have any orientation in early-type systems. In order to check whether our sample is biased toward Seyfert 1's in late-type galaxies and toward Seyfert 2's in early-type galaxies, we show in Figure 3 a histogram of their morphological types, where the parameter $T=-4$ corresponds to elliptical galaxies, the earliest morphological type, and $T=5$ corresponds to Sc's, the latest morphological type. The group distributions are almost equal, the only difference being the existence of some Seyfert 2's with $T \leq-2$ (as early as S0) without any corresponding Seyfert 1's in this region of the diagram. These objects cannot be distinguished in the position angle histograms, assuring us that the groups are not biased by different morphological types.

We also checked for a trend for the obscuring torus axis to align along the minor axis in late-type galaxies. In Figure 4 , we show a plot of the morphological type versus $\Delta$ P.A. There is no systematic trend for late-type objects to have large $\Delta$ P.A. values, which means that their radio structures are not preferentially aligned with the host galaxy minor axis. This result suggests that the trend found by Wilson \& Tsvetanov (1994) was most likely due to the small number of objects in their sample.

\section{DISCUSSION}

The results presented in Figure 1 can be interpreted from the point of view of the scheme proposed by Schmitt \& Kinney (1996), in which the Seyfert 1's have their torus axis aligned preferentially close to the galaxy plane axis while the Seyfert 2's have their torus axis aligned at an intermediate angle between the galaxy plane and its axis. Here we describe a simple model, developed in order to study the orientation of the projected radio structure (assumed to be aligned with the torus axis) due to its inclination relative to the line of sight. In our model, we assume a uniform distribution of orientation angles between the obscuring torus axis and the host galaxy plane axis. Then we predict what the observed values for $\Delta$ P.A. would be, given that uniform distribution.

Figure 5 shows a schematic representation of our model. In this figure, the galaxy disk lies on the $X-Y$ plane, and the elongated radio structure, represented by the unit vector $\hat{\boldsymbol{k}}_{j}$, makes an angle $\beta$ with the $Z$-axis (galaxy plane axis). The elongated radio structure is equally likely to be in any position along the circle $\mathrm{C}$, which is described by the azimuthal angle $\theta$, measured from the $X$ - to the $Y$-axis. The galaxy is inclined relative to the line of sight $\left(Z^{\prime}\right.$-axis) by an angle $i$, such that the inclined galaxy major axis is coincident with the $X$-axis.

In this model the angle $\beta$, between the radio axis and the galaxy plane axis, assumes values in the range $1^{\circ} \leq \beta \leq 90^{\circ}$ and is varied in steps of $1^{\circ}$. For each value of $\beta$, we generate 1000 vectors equally spaced along one-half the circle C. As a result of the symmetry of the problem, the angle $\theta$ is varied only in the range $-90^{\circ} \leq \theta \leq 90^{\circ}$. Also, because of the fact that the circle $\mathrm{C}$ describes different perimeters for different values of $\beta$, the results were weighted, for each value of $\beta$, by the area of a ring of $1^{\circ}$ described by the circle $C$ on the surface of a sphere, relative to the whole sphere area. This is necessary in order to avoid an oversampling in the number of vectors for smaller $\beta$-values, relative to larger ones. The galaxy inclination $i$ is then varied over the range $15^{\circ} \leq i \leq 60^{\circ}$ in steps of $1^{\circ}$. We chose this range because for $i<15^{\circ}$ it is difficult to measure the galaxy inclination angle and it is considered to be face-on while, for $i>60^{\circ}$, there is only a small number of galaxies with such inclination in our sample. The small number of objects with $i>60^{\circ}$ suggests

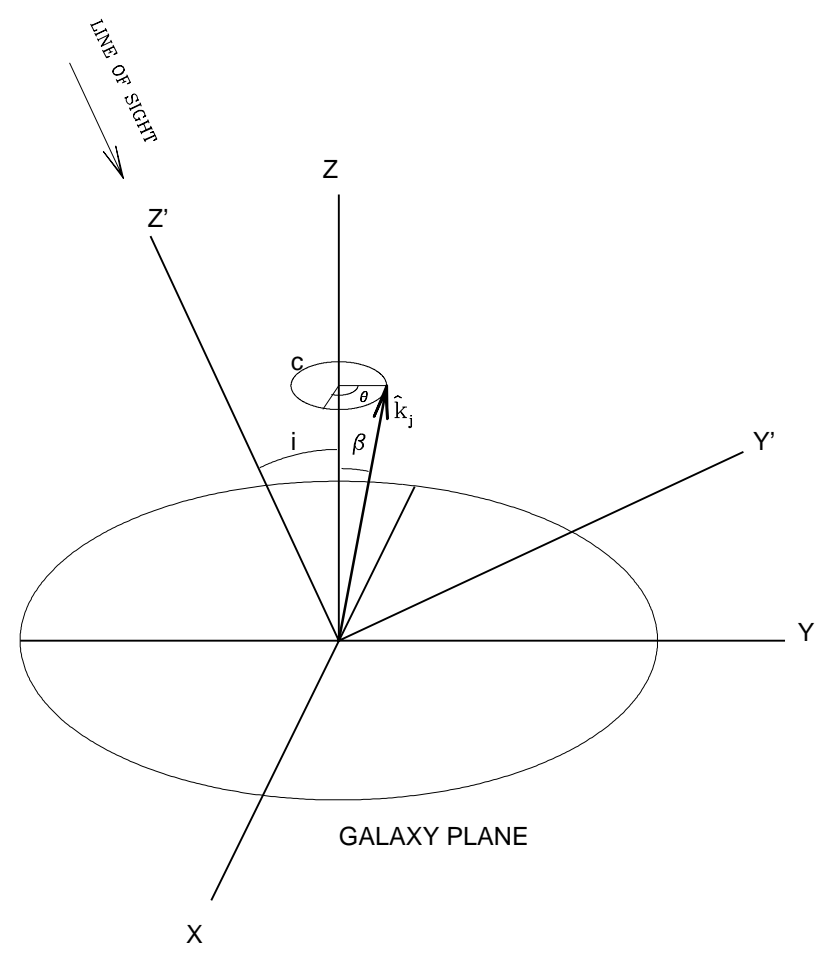

FIG. 5.-Our radio "jet" model. The host galaxy disk lies in the $X-Y$ plane, and the radio "jet" is represented by the vector $\hat{\boldsymbol{k}}_{j}$, which makes an angle $\beta$ with the $Z$-axis (galaxy plane axis) and is likely to be in any direction along the circle $\mathrm{C}$, which is described by the angle $\theta$. The angle $i$ describes the galaxy inclination relative to the line of sight. 


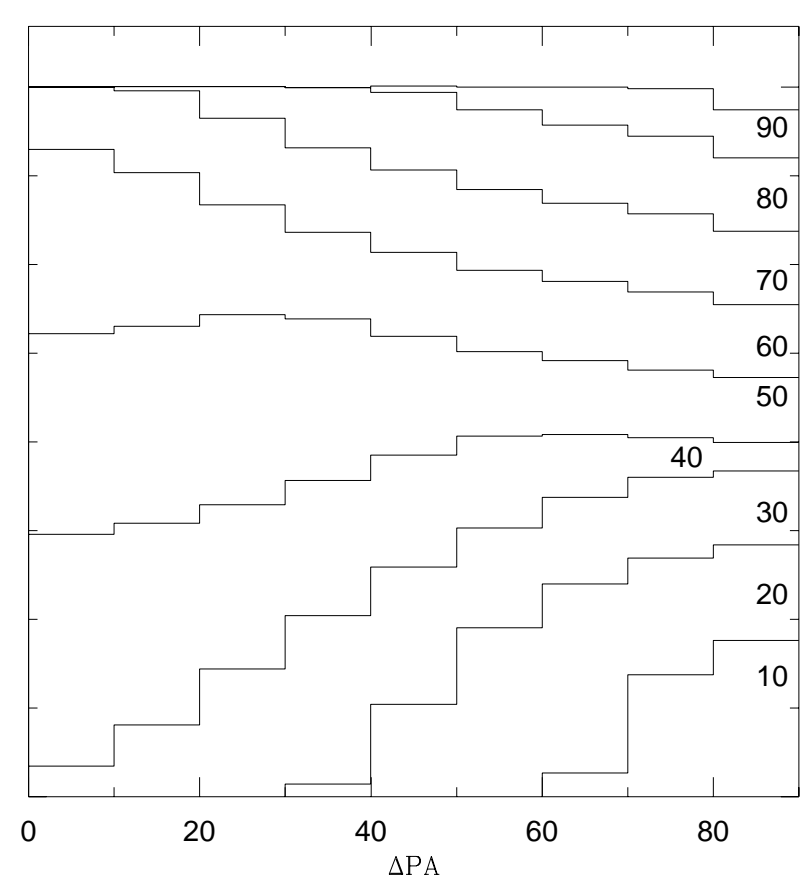

FIG. $6 a$

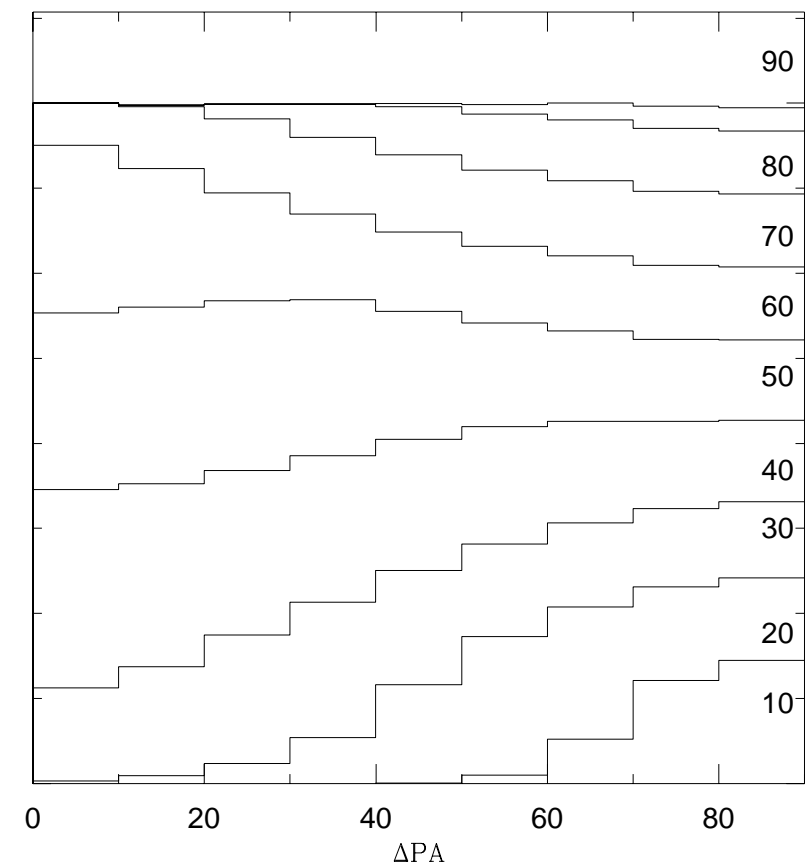

FIG. $6 b$

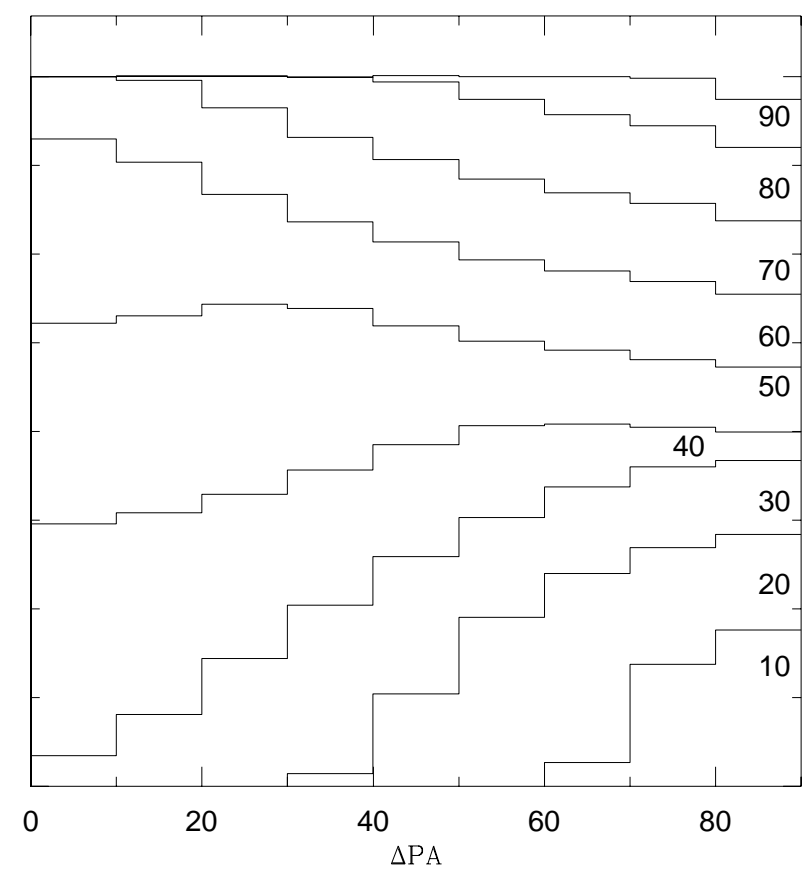

FIG. $6 c$

FIG. 6.-Results of the models. The lines are the cumulative sum of all the vectors with $\beta$ less than or equal to the value indicated below the line. (a) Seyfert 1's with $r>0.4 ;(b)$ Seyfert 1's with $r>0.4 ;(c)$ Seyfert 2's.

an inclination-dependent selection bias. We have also tested the model for smaller and larger values of $i$, but the final result does not change.

The projected components of each individual radio jet vector (for every $\theta-, \beta$-, and $i$-value), in the directions $X, Y^{\prime}$, and $Z^{\prime}$, can be calculated with the following relations:

$$
P_{X}=\cos \theta \sin \beta,
$$

$P_{Y^{\prime}}=\cos i \sin \theta \sin \beta+\sin i \cos \beta$,

$P_{Z^{\prime}}=\cos i \cos \beta-\sin i \sin \theta \sin \beta$.
The observed difference between the elongated radio structure P.A. and the major-axis P.A. ( $\Delta$ P.A.) is given by

$$
\Delta \text { P.A. }=\arctan \left(P_{Y^{\prime}} / P_{X}\right) .
$$

We divide $\Delta$ P.A. into $10^{\circ}$ bins and count the number of vectors with projected $\Delta$ P.A. inside each bin, for a given $\beta$-value. This number, normalized to the total number of vectors, can be considered to be the probability of an elongated radio structure, making an angle $\beta$ with the host 


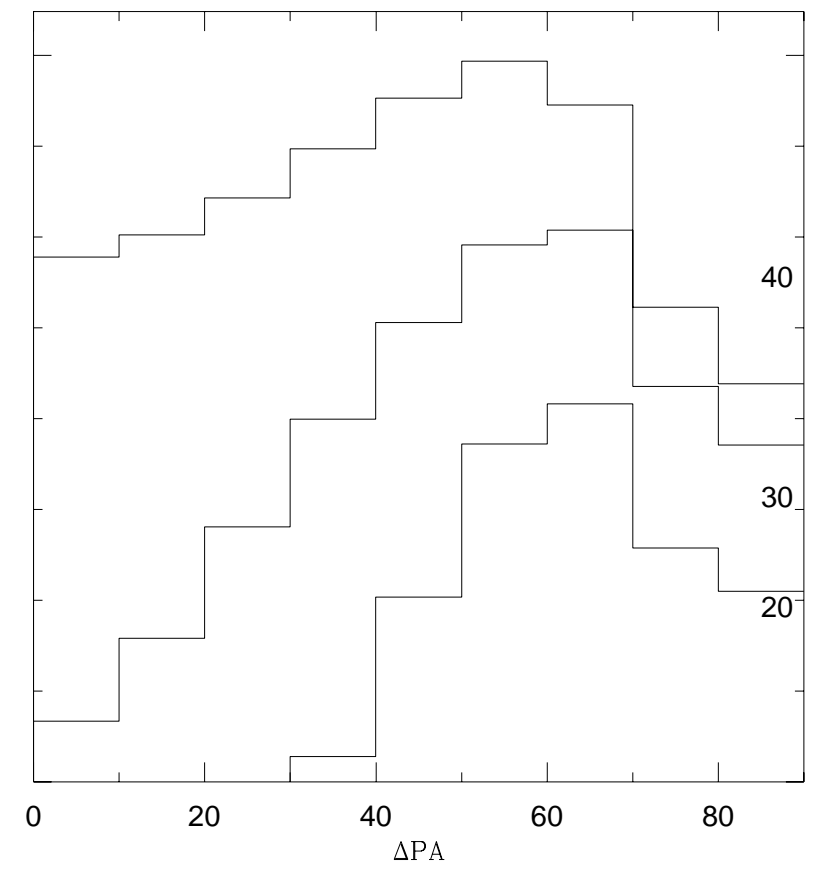

FIG. $7 a$

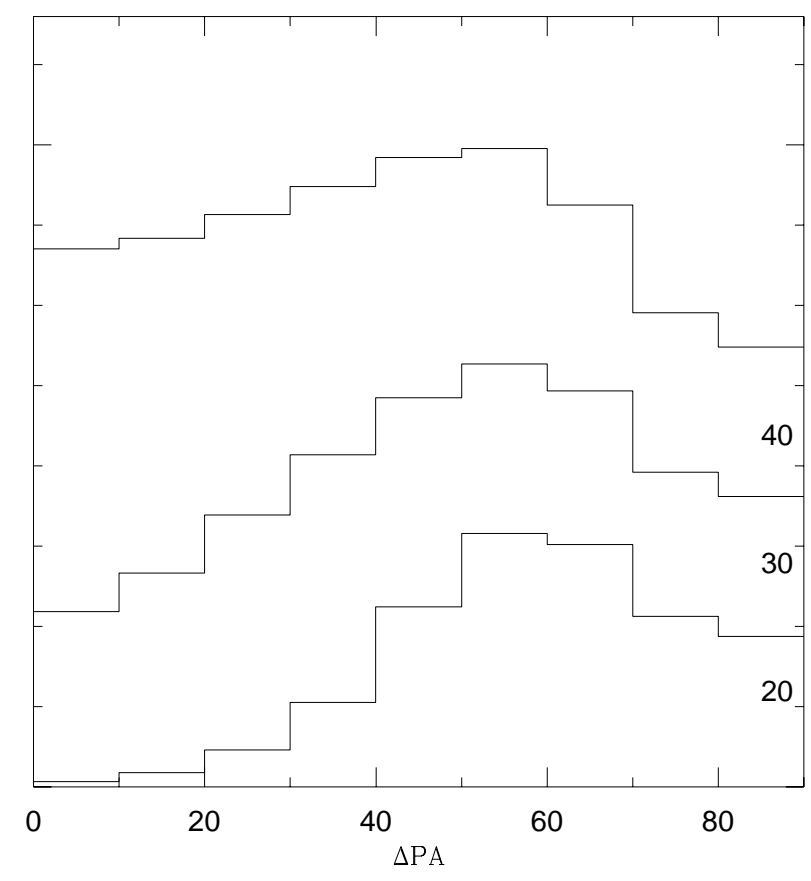

FIG. $7 b$

FIG. 7.-Results of the Seyfert 1 models excluding the cases with $\beta \leq 10^{\circ}$. (a) $r>0.4$; (b) $r>0.2$.

galaxy axis, being seen at a projected angle $\Delta$ P.A. from the galaxy major axis, considering inclination effects.

The model also includes some constraints. First, a galaxy is only considered to be a Seyfert 1 if the angle between $Z^{\prime}$ and $\hat{\boldsymbol{k}}_{j}$ is less than $30^{\circ}$, corresponding to observing into the opening of the torus; otherwise it is a Seyfert 2. This number was obtained from Osterbrock \& Shaw (1988) and corresponds to half the torus opening angle. Second, if the projected length $(r)$ of the unit vector $\hat{\boldsymbol{k}}_{j}$ in the $X-Y^{\prime}$ plane is less than a given value, it is assumed to be unresolved and is not considered when we count the number of vectors that fall inside different $\Delta$ P.A. bins. This corresponds to the case in which the elongated radio structure is observed pole-on and is therefore not observed as elongated. Since we do not know the intrinsic length of the elongated radio structure, we note that they usually have sizes on the order of $1^{\prime \prime}-2^{\prime \prime}$ and that the VLA resolution at $6 \mathrm{~cm}$ in the A configuration is $\approx 0$ ". 4 . We thus study two cases, $r>0.2$ and $r>0.4$. Note also that Seyfert 2's are not affected by the projected size restriction, because the vectors with angle between $Z^{\prime}$ and $\hat{\boldsymbol{k}}_{\boldsymbol{j}}$ greater than $30^{\circ}$ will have $r>0.5$.

Figure 6 shows the model results. The individual lines are cumulative histograms, representing the sum over all $\beta$ angles less than or equal to the value indicated at the right and below each line. The vertical axes of these plots are normalized relative to the $\Delta$ P.A. bin with the largest number of vectors on the $\beta=90^{\circ}$ bin. Each histogram can be considered as the cumulative probability of an elongated radio structure, making an angle less than or equal to $\beta$, being seen at a projected angle $\Delta$ P.A. from the galaxy major axis. In Figure $6 a$, we show the results for Seyfert 1's with projected length $(r)$ larger than 0.4 . Figure $6 b$ shows the results for Seyfert 1's with projected length larger than 0.2, and Figure $6 c$ shows the results for Seyfert 2's. The $\Delta$ P.A. distributions for Seyfert 1's are very similar, independent of the $r$-value. For small $\beta$-values, there is a larger probability of observing an elongated radio structure closer to the host galaxy minor axis (large $\Delta$ P.A.). For larger $\beta$-values, the probability of observing the elongated radio structure closer to the major axis increases. When we consider the distribution for all $\beta$-angles together (the $90^{\circ}$ line), the probability of finding a vector at any $\Delta$ P.A. bin is approximately the same. The distribution of $\Delta$ P.A.'s for Seyfert 2's is similar to that for Seyfert 1's. However, the probability of finding small $\Delta$ P.A.'s, which correspond to an elongated radio structure close to the host galaxy major axis, only increases when we go to larger $\beta$-values relative to those of Seyfert 1's.

Comparing the model results from Figure 6 with the observed $\Delta$ P.A.'s in Figure 1, we see that the lack of Seyfert 1 galaxies with small $\Delta$ P.A. values can only be understood if there are very few Seyfert 1 galaxies in which the angle between the elongated radio structure and the galaxy plane axis $(\beta$ in the model $)$ is large $\left(\beta>30^{\circ}-40^{\circ}\right)$. Similarly, when we compare the results for the Seyfert 2 models with the observed values, the distribution of $\Delta$ P.A. values can be understood if the Seyfert 2 galaxies can take on any value for the angle between the elongated radio structure and the galaxy plane axis.

These results support the scheme proposed by Schmitt \& Kinney (1996). However, that scheme cannot explain the lack of both Seyfert 1's and Seyfert 2's with large $\Delta$ P.A. values, which would correspond to elongated radio structures aligned with the host galaxy minor axis. This lack of large $\Delta$ P.A. values suggests a physical zone of avoidance, where for some reason the radio axis is never closely aligned intrinsically with the galaxy plane axis. If we consider a flat distribution of $\Delta$ P.A.'s, we calculate that, for our sample of 46 galaxies, we would expect 10 galaxies with $\Delta$ P.A. $>70^{\circ}$. Considering a sample of only 31 galaxies, which corresponds to the number of Seyfert 2's in our sample, we would expect seven galaxies with $\Delta$ P.A. $>70^{\circ}$. From Poisson sta- 
tistics we calculate that the a posteriori probability of observing only one galaxy with $\Delta$ P.A. $>70^{\circ}$, when the expected number is 10 , is $10^{-4.78}$, or $10^{-3.47}$ for the case in which seven galaxies are expected.

There may be some effect, which we are not taking into account, that makes large $\Delta$ P.A. unobservable. One possible solution for the lack of Seyfert 1's with elongated radio structures aligned with the host galaxy minor axis could be that, when the obscuring torus is closely aligned with the host galaxy axis, an elongated radio structure is not produced. As a result of the low $\mathrm{H}$ I density in the central regions of spiral galaxies (Rots 1975; Begeman 1988), the radio "jet" would not interact with a large enough quantity of matter and consequently would not radiate enough to be detected. Alternatively, these nuclear disks in the same plane as the host galaxy may have fewer dissipation/fueling mechanisms. Yet another possibility is that the nuclear axes reflect a past triggering merger and that the approaches of companions are statistically anisotropic. To incorporate such effects, we show in Figures $7 a$ and $7 b(r>0.4$ and $r>0.2$, respectively) the models for Seyfert 1's with $\beta \leq 10^{\circ}$ excluded. In this case the probability of observing an elongated radio structure with $\Delta$ P.A. $>70^{\circ}$ is smaller than for values of $\Delta$ P.A. $<70^{\circ}$. This makes the observed and modeled $\Delta$ P.A. distributions look much more alike, although we would still expect to detect some objects with $\Delta$ P.A. $>70^{\circ}$. While this solution can perhaps solve the problem for Seyfert 1's, it does not suffice for Seyfert 2's, for which the statistics are better. A simple visual inspection of Figure $6 c$ reveals that the exclusion of all vectors with $\beta \leq 10^{\circ}$ does not change the distribution of $\Delta$ P.A.'s significantly. Even if we exclude all vectors with $\beta \leq 30^{\circ}$, the probability of observing a Seyfert 2 with $\Delta$ P.A. $>70^{\circ}$ would be smaller, but it would not explain the deficit that we observe.

The lack of Seyfert 1 galaxies with elongated radio structures aligned with the galaxy major axis is consistent with the results obtained by Keel (1980) and Maiolino \& Rieke (1995). These authors showed that there is a deficiency of edge-on Seyfert 1 galaxies and that Seyfert types 1.8 and 1.9 are more likely to be in edge-on galaxies. These results suggest that in the case of edge-on objects we could be observing the nuclear region directly, through the polar region of the nuclear torus, but not seeing the broad-line region because of shadowing by gas and dust in the galaxy disk.

On the other hand, our results differ from those of Colbert et al. (1996) and Baum et al. (1993). These authors found that the large-scale $(\approx 1 \mathrm{kpc})$ radio structure of Seyfert galaxies are preferentially aligned with the host galaxy minor axis. Baum et al. (1993) also compared the small-scale radio structures with the large-scale radio struc- tures and found that their P.A.'s are different. Colbert et al. (1996) suggest that the small-scale radio jets are possibly diverted by dense molecular clouds, like the scenario proposed for NGC 1068 by Gallimore, Baum, \& O’Dea (1996), and then generate the large-scale radio structures that we see.

\section{SUMMARY AND CONCLUSIONS}

We have shown in this paper that there is a lack of Seyfert 1 galaxies with radio structures aligned with the host galaxy major axis while, for Seyfert 2's, the radio structures are oriented along almost any direction in the galaxy. Both groups also show a deficiency of objects with elongated radio structures closely aligned with the host galaxy plane axis.

We developed a model to calculate the distribution of $\Delta$ P.A., the difference between the orientation of the radio axis and host galaxy major axis, based on the assumption that the angle between the radio axis and the host galaxy plane axis $(\beta)$ is uniformly distributed between $0^{\circ}$ and $90^{\circ}$, taking into account the effect of the galaxy inclination and resolution of the elongated radio structure. From a comparison of the observed $\Delta$ P.A. distribution of Seyfert 1's with the distribution predicted by the model, we can explain the small number of objects with small $\Delta$ P.A.'s only if their torus axes lie closer than $\approx 30^{\circ}$ to the host galaxy axis. The lack of Seyfert 1's with $\Delta$ P.A. $>70^{\circ}$ can be partially explained if we assume that the elongated radio structures are not often produced closer than $\approx 10^{\circ}$ to the host galaxy plane axis, and several possible explanations for this are suggested. For the Seyfert 2's, the observed distribution can be explained if the torus axis assumes any angle relative to the host galaxy plane, with the exception of the region with $\Delta$ P.A. $>70^{\circ}$.

These results, together with those from Keel (1980) and Maiolino \& Rieke (1995), showing that Seyfert 1's are less likely to be found in edge-on galaxies, as well as the results from Lawrence \& Elvis (1982) and de Zotti \& Gaskell (1985), showing that the broad-line region in edge-on galaxies is reddened, are in good agreement with the model proposed by Schmitt \& Kinney (1996). The paucity of objects showing close alignment between radio axis and galaxy plane axis remains unexplained.

This work was supported by NASA under grant AR05810.01-94A and by the Brazilian institution CNPq. This research has made use of the NASA/IPAC Extragalactic Database (NED), which is operated by the Jet Propulsion Laboratory, Caltech, under contract with NASA. We also would like to thank E. Colbert for useful discussions.

\section{REFERENCES}

Antonucci, R. R. J. 1993, ARA\&A, 31, 473

Antonucci, R. R. J., \& Miller, J. S. 1985, ApJ, 297, 621

Baum, S. A., O’Dea, C. P., Dallacasa, D., de Bruyn, A. G., \& Pedlar, A. 1993, ApJ, 419, 553

Begeman, K. 1988, Ph.D. thesis, Univ. Groningen

Bower, G., Wilson, A. S., Morse, J. A., Gelderman, R., Whittle, M., \& Mulchaey, J. S. 1995, ApJ, 454, 106

Colbert, E. J. M., Baum, S. A., Gallimore, J. F., O’Dea, C. P., \& Christensen, J. A. 1996, ApJ, 467, 551

de Vaucouleurs, G., de Vaucouleurs, A., Corwin H. G., Jr., Buta, R. J.,

Paturel, G., \& Fouqué, P. 1991, Third Reference Catalogue of Bright

Galaxies (New York: Springer)

de Zotti, G., \& Gaskell, C. M. 1985, A\&A, 147, 1

Edelson, R. A. 1987, ApJ, 313, 651

Gallimore, J. F., Baum, S. A., \& O’Dea, C. P. 1996, ApJ, 464, 198

Keel, W. C. 1980 , AJ, 85, 198

Kinney, A. L., Antonucci, R. R. J., Ward, M. J., Wilson, A. S., \& Whittle, M. 1991, ApJ, 377, 100

Kukula, M. J., Pedlar, A., Baum, S. A., \& O’Dea, C. P. 1995, MNRAS, 276, 1262

Lawrence, A., \& Elvis, M. 1982, ApJ, 256, 410

Maiolino, R., \& Rieke, G. H. 1995, ApJ, 454, 95

Meurs, E. J. A., \& Wilson, A. S. 1984, A\&A, 136, 206

Miller, J. S., \& Goodrich, R. W. 1990, ApJ, 355, 456

Miyaji, T., Wilson, A. S., \& Pérez-Fournon, I. 1992, ApJ, 385, 137

Morris, S., Ward, M., Whittle, M., Wilson, A. S., \& Taylor, K. 1985, MNRAS, 216, 193

Osterbrock, D. E., \& Shaw, R. A. 1988, ApJ, 327, 89

Pogge, R. W. 1989, ApJ, 345, 730

Rots, A. H. 1975, A\&A, 45, 43 
Schmitt, H. R \& Kinney, A. L. 1996, ApJ, 463, 498

Ulvestad, J. S., \& Wilson, A. S. 1984a, ApJ, 278, 544 1984 b, ApJ, 285, 439

Ulvestad, J. S., Wilson, A. S., \& Sramek, R. A. 1981, ApJ, 247, 419

Unger, S. W., Lawrence, A., Wilson, A. S., Elvis, M., \& Wright, A. E. 1987, MNRAS, 228, 521
Urry, C. M., \& Padovani, P. 1995, PASP, 107, 803

Weaver, K., Wilson, A. S., \& Baldwin, J. A. 1991, ApJ, 366, 50

Wilson, A. S., \& Tsvetanov, Z. I. 1994, AJ, 107, 1227

Wilson, A. S., Ward, M. J., \& Haniff, C. A. 1988, ApJ, 334, 121

Wilson, A. S., \& Willis, A. G. 1980, ApJ, 240, 429 